\title{
Regular Hexagons Associated to Triangles with Equal Centroids
}

\author{
Zvonko Čerin
}

Zvonko Čerin, born 1950 in Zagreb, began his studies at the University of Zagreb. The years from 1973 to 1976 he spent at the Louisiana State University in Baton Rouge where he received his $\mathrm{PhD}$. He has published numerous papers on various topics in topology and is now Professor at the University of Zagreb. His recent interest in geometry began under the influence of Professor Baldi from the Università di Torino who introduced him to the world of computers. In his spare time he likes to play rather competitive recreational tennis.

\section{Introduction}

Let $r$ denote a real number. For points $A$ and $B$, let $r_{A}^{B}$ be the point $A$ when $A=B$, the point $B$ when $A \neq B$ and $r=-1$, and the point $P$ on the line $A B$ such that $|A P| /|P B|=r$ when $A \neq B$ and $r \neq-1$.

In Problem 1493 in the February 1996 issue of Mathematics Magazine, Jiro Fukuta observed the following method of generating regular hexagons associated with any triangle $A B C$.

Fukuta's Method. Let $0 \leq r<1$. Let $P_{1}=r_{B}^{C}, P_{2}=r_{C}^{B}, P_{3}=r_{C}^{A}, P_{4}=r_{A}^{C}, P_{5}=r_{A}^{B}$, and $P_{6}=r_{B}^{A}$. Let $P_{7}=P_{1}$. Let $Q_{i} P_{i} P_{i+1},(i=1,2, \ldots, 6)$, be the equilateral triangles built outwards on the sides of the hexagon $P_{1} P_{2} \cdots P_{6}$. Let $Q_{0}=Q_{6}$ and $Q_{7}=Q_{1}$. Let $F_{i}$ be the centroid of the triangle $Q_{i-1} Q_{i} Q_{i+1}, i=1,2, \ldots, 6$. Then $F_{1} F_{2} \cdots F_{6}$ is a regular hexagon whose center coincides with the centroid of the triangle $A B C$.

Geometrische Konstruktionen, die auf eine reguläre Figur führen, üben einen besonderen ästhetischen Reiz aus. Zvonko Čerin liefert uns hier ein Beispiel. Er gibt sich zwei reelle Zahlen $p$ und $q$ vor und zwei Dreiecke mit gemeinsamem Schwerpunkt $S$. Ausgehend von einander entsprechenden Ecken der beiden Dreiecke führt er eine Anzahl von Konstruktionsschritten durch, die im einzelnen von $p$ und $q$ abhängig sind. Es resultiert schliesslich ein Sechseck. Und siehe da: Das Sechseck ist regulär und besitzt den Mittelpunkt S. ust 
The solution by O.P. Lossers from the Technical University in Eindhoven (The Netherlands) has appeared in the February 1997 issue. His solution uses complex numbers and is about two printed pages long. The aim of this note is to propose the following more general method of creating regular hexagons associated with two triangles with common centroid.

New Method. Let $A B C$ and $X Y Z$ be two triangles with common centroid, and let $p$ and $q$ be any real numbers. Let $P_{1}=p_{A}^{X}, P_{2}=q_{X}^{A}, P_{3}=p_{B}^{Y}, P_{4}=q_{Y}^{B}, P_{5}=p_{C}^{Z}$, and $P_{6}=q_{Z}^{C}$. Let $P_{7}=P_{1}$. For $i=1,2, \ldots, 6$, let $Q_{i} P_{i} P_{i+1}$ be the six equilateral triangles built on the sides of the hexagon $P_{1} P_{2} \cdots P_{6}$ all with the same orientation. Let $Q_{0}=Q_{6}$ and $Q_{7}=Q_{1}$. Let $G_{i}$ be the centroid of the triangle $Q_{i-1} Q_{i} Q_{i+1}, i=1,2, \ldots, 6$. Then $G_{1} G_{2} \cdots G_{6}$ is a regular hexagon whose center coincides with the centroid of the triangle $A B C$.

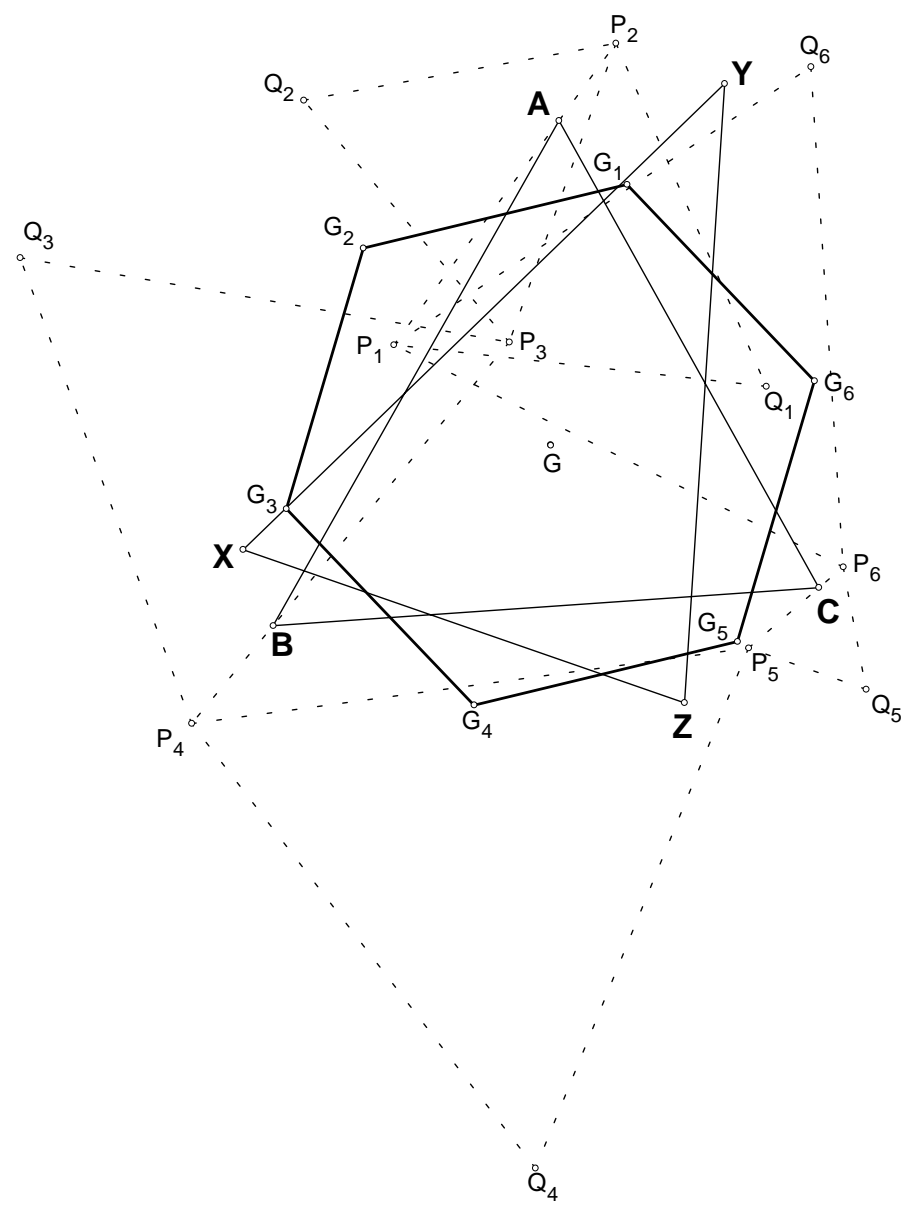

Fig. 1 The New Method: General situation. 
Observe that there is no condition on the numbers $p$ and $q$ and that there are two regular hexagons obtained in this way because there are two orientation classes. Moreover, the new method reduces to Fukuta's method if we consider a pair $A B C$ and $B C A$ and put $p=q=r$.

There are many triangles associated with the triangle $A B C$ which have the same centroid. For example, the complementary triangle $A_{m} B_{m} C_{m}$ whose vertices are midpoints of sides, the anticomplementary triangle $A_{a} B_{a} C_{a}$ whose vertices are intersections of parallels through vertices to opposite sides, the first Brocard triangle $A_{b} B_{b} C_{b}$ of $A B C$, the triangles whose vertices are vertices (centers) of equilateral triangles built on sides either outwards or inwards, and the degenerate triangle $G G G$, where $G$ is the centroid of $A B C$.

\section{Verification of the new method}

In our proof we shall work in the complex plane, and therefore we shall allow ourselves to regard points as complex numbers. In the references we have listed several books that give nice introductions into this technique of proof in geometry.

For an expression $E x$ that depends on the set of variables $S$ and a permutation $b$ of $S$ let $b(E x)$ denote the expression obtained by permuting the variables according to $b$. For example, if $E x=3 u v^{5} w^{3}$ and $b(u)=v, b(v)=w$, and $b(w)=u$, then $b(E x)=3 u^{3} v w^{5}$.

Let $s$ and $t$ denote the substitutions $\{A=B, B=C, C=A, X=Y, Y=Z, Z=X\}$ and $\{A=C, B=A, C=B, X=Z, Y=X, Z=Y\}$. We prove the following amusing theorem which could be regarded as the main fact implying the new method.

Theorem 1. Let the triangles $A B C$ and $X Y Z$ have the common centroid $G$. Let $P_{1}=A$, $P_{2}=X, P_{3}=B, P_{4}=Y, P_{5}=C$, and $P_{6}=Z$. Let the points $G_{1}, G_{2}, \ldots, G_{6}$ be constructed as in the description of the new method. Then $G_{1} G_{2} \cdots G_{6}$ is a regular hexagon with center $G$.

Proof. Without loss of generality, we can assume that $G$ is the origin of the complex plane, so that the common centroid of $A B C$ and $X Y Z$ corresponds to the complex number zero. It follows that $A+B+C=0$ and $X+Y+Z=0$.

Set $\Omega=\frac{1}{2}+i \frac{\sqrt{3}}{2}$ and $\omega=\frac{1}{2}-i \frac{\sqrt{3}}{2}$. Then $\omega$ is the complex conjugate of $\Omega$, and one has $\Omega=1-\omega, \Omega^{2}=-\omega$, and $\Omega \omega=1$. It follows that $Q_{1}=\Omega A+\omega X$ and $Q_{2}=\Omega X+\omega B$ while $Q_{3}=s\left(Q_{1}\right), Q_{4}=s\left(Q_{2}\right), Q_{5}=t\left(Q_{1}\right)$, and $Q_{6}=t\left(Q_{2}\right)$. Also,

$$
G_{1}=\frac{1}{3}(\Omega A+B+X+\omega Y), \quad G_{2}=\frac{1}{3}(\Omega X+Y+B+\omega C)
$$

$G_{3}=s\left(G_{1}\right), G_{4}=s\left(G_{2}\right), G_{5}=t\left(G_{1}\right)$, and $G_{6}=t\left(G_{2}\right)$.

Since

$$
\Omega G_{1}=\frac{1}{3}(-\omega A+\Omega B+\Omega X+Y)=\frac{1}{3}(\Omega X+Y+B+\omega(-A-B))=G_{2},
$$

and similarly $\Omega G_{2}=G_{3}, \Omega G_{3}=G_{4}, \Omega G_{4}=G_{5}, \Omega G_{5}=G_{6}$, and $\Omega G_{6}=G_{1}$, we conclude that the points $G_{1}, \ldots, G_{6}$ are the vertices of a regular hexagon with center at the centroid of $A B C$ and $X Y Z$ because rotating by $\frac{\pi}{3}$ around the origin maps the point $G_{i}$ to a point $G_{i+1}$ for $1 \leq i<6$ and $G_{6}$ to $G_{1}$. 


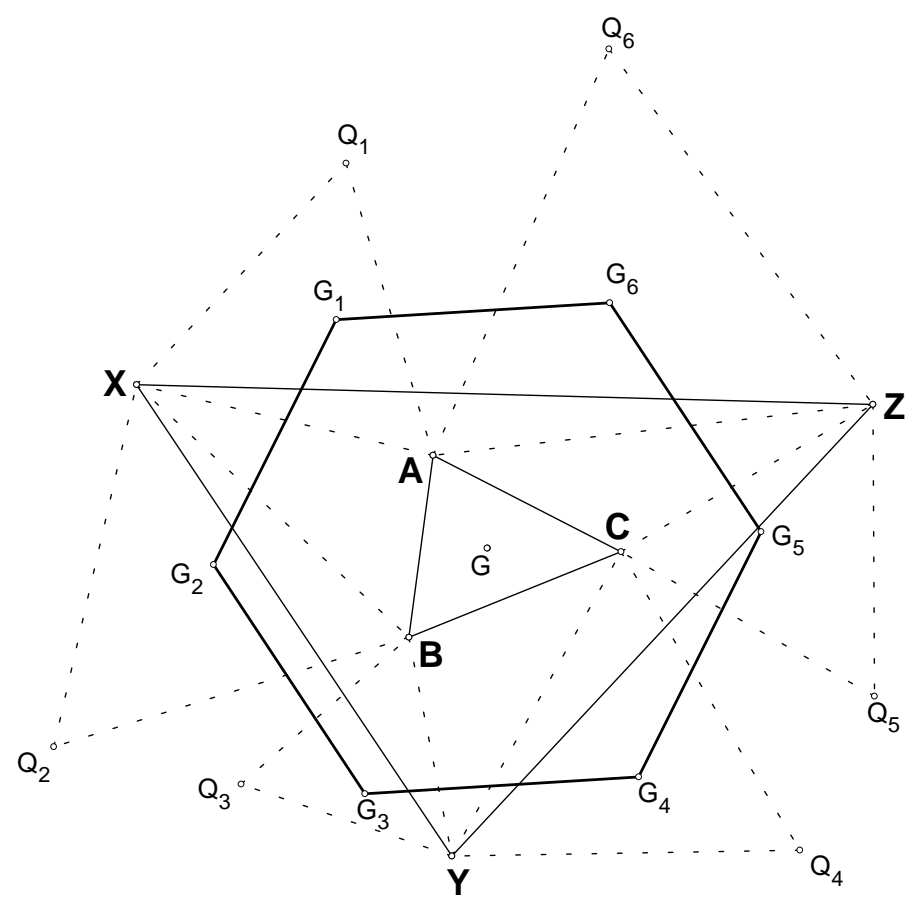

Fig. 2 In Theorem 1 we build equilateral triangles on the sides of the hexagon $A X B Y C Z$

In the above calculations we have assumed that the triangle $\omega \Omega J$ is positively oriented, where $J$ is the point corresponding to the complex number -1 , and that we have constructed on the sides of the hexagon $P_{1} P_{2} \cdots P_{6}$ six positively oriented equilateral triangles. We obtain similar conclusions in the situation when negatively oriented triangles are used.

The fact that $G_{1} G_{2} \cdots G_{6}$ is a regular hexagon can also be proved using the following idea. Assuming that $G$ is the origin of the complex plane, it is sufficient to show that (i) the triangles $G_{1} G_{3} G_{5}$ and $G_{2} G_{4} G_{6}$ are symmetric to each other with respect to the origin, (ii) the triangle $G_{1} G_{3} G_{5}$ is equilateral, and (iii) its centroid is the origin. Each of these three assertions can be proved in a few lines.

The new method follows from the Theorem 1 and the following result.

Theorem 2. Let the triangles $A B C$ and $X Y Z$ have the common centroid $G$, and let $r$ be any real number. Let $P=r_{A}^{X}, Q=r_{B}^{Y}$, and $R=r_{C}^{Z}$. Then $G$ also is the centroid of the triangle $P Q R$.

Proof. Under the same simplification as in the proof of the Theorem 1 , for $r \neq-1$, we have $P=q(A+r X), Q=q(B+r Y)$, and $R=q(C+r Z)$, where $q=1 /(r+1)$. The centroid of the triangle $P Q R$ is $q(A+B+C+r(X+Y+Z)) / 3$. But, this number is zero because $A+B+C=0$ and $X+Y+Z=0$. Hence, the centroid of $P Q R$ is the origin. For $r=-1$, the proof is trivial. 
Here are two more results analogous to the Theorem 2 that give us new methods for selecting points $P_{1}, P_{2}, \ldots, P_{6}$. They use the following notion of a $(p, q)$-point of a triangle $A B C$.

Let $p$ and $q$ be any real numbers. Let $(p, q)_{A B C}$ be a short notation for the point $p_{A}^{W}$, where $W=q_{B}^{C}$. Notice that $(1,1)_{A B C}$ is the centroid of $A B C$.

Theorem 3. Let $p$ and $q$ be any real numbers. Let $X=(p, q)_{A B C}, Y=(p, q)_{B C A}$, and $Z=(p, q)_{C A B}$. Then the triangles $A B C$ and $X Y Z$ have the same centroid.

Proof. Let $f=1 /(p+1)$ and $g=1 /(q+1)$. Obviously we have $X=f A+f g p B+$ $f g p q C, Y=s(X)$, and $Z=t(X)$. It follows that the triangle $X Y Z$ has the centroid at the complex number $(A+B+C) / 3$. Hence, $A B C$ and $X Y Z$ have the same centroid. This proof assumes that both $p$ and $q$ are different from -1 . In these exceptional cases the proof is even simpler, and we therefore leave it to the reader.

Theorem 4. Let $p$ and $q$ be any real numbers, and let $A B C$ and $X Y Z$ be triangles with the common centroid $G$. Let $P=(p, q)_{A Y Z}, Q=(p, q)_{B Z X}$, and $R=(p, q)_{C X Y}$. Then $G$ also is the centroid of the triangle $P Q R$.

Proof. We can assume that $G$ is the origin. Then $P=f A+f g p Y+f g p q Z, Y=s(X)$, and $Z=t(X)$, where $f=1 /(p+1)$ and $g=1 /(q+1)$. It follows that the triangle $P Q R$ has centroid $(A+B+C+p(X+Y+Z)) / 3$. Hence, $P Q R, A B C$ and $X Y Z$ have the same centroid. This proof again assumes that both $p$ and $q$ are different from -1 . The proof for the cases, when this is not true is easy, and left to the reader.

\section{Diagonal method}

In this section we shall describe another method of associating a regular hexagon with two triangles having the same centroid. This time the points $Q_{1}, Q_{2}, \ldots, Q_{6}$, instead of being the vertices of the equilateral triangles built on sides of $P_{1} P_{2} \cdots P_{6}$, are the centers of equilateral triangles built on small diagonals of $P_{1} P_{2} \cdots P_{6}$.

Diagonal Method. Let $A B C$ and $X Y Z$ be two triangles with common centroid, and let $p$ and $q$ be any real numbers. Let $P_{1}=p_{A}^{X}, P_{2}=q_{X}^{A}, P_{3}=p_{B}^{Y}, P_{4}=q_{Y}^{B}, P_{5}=p_{C}^{Z}$, and $P_{6}=q_{Z}^{\mathrm{C}}$. Let $P_{7}=P_{1}$ and $P_{8}=P_{2}$. For $i=1,2, \ldots, 6$, let $Q_{i}$ be the centroids of the the six equilateral triangles built on the small diagonals $P_{i} P_{i+2}$ of the hexagon $P_{1} P_{2} \cdots P_{6}$ all with the same orientation. Let $Q_{0}=Q_{6}$ and $Q_{7}=Q_{1}$. Let $D_{i}$ be the centroid of the triangle $Q_{i-1} Q_{i} Q_{i+1}, i=1,2, \ldots, 6$. Then $D_{1} D_{2} \cdots D_{6}$ is a regular hexagon whose center coincides with the centroid of the triangle $A B C$.

This method is a simple consequence of the following theorem.

Theorem 5. Let $A B C$ and $X Y Z$ be two triangles with common centroid $G$. Let $P_{1}=A$, $P_{2}=X, P_{3}=B, P_{4}=Y, P_{5}=C$, and $P_{6}=Z$. Let points $D_{1}, D_{2}, \ldots, D_{6}$ be constructed as above from centroids of equilateral triangles built on small diagonals of $P_{1} P_{2} \cdots P_{6}$. Then $D_{1} D_{2} \cdots D_{6}$ is a regular hexagon with center $G$. 


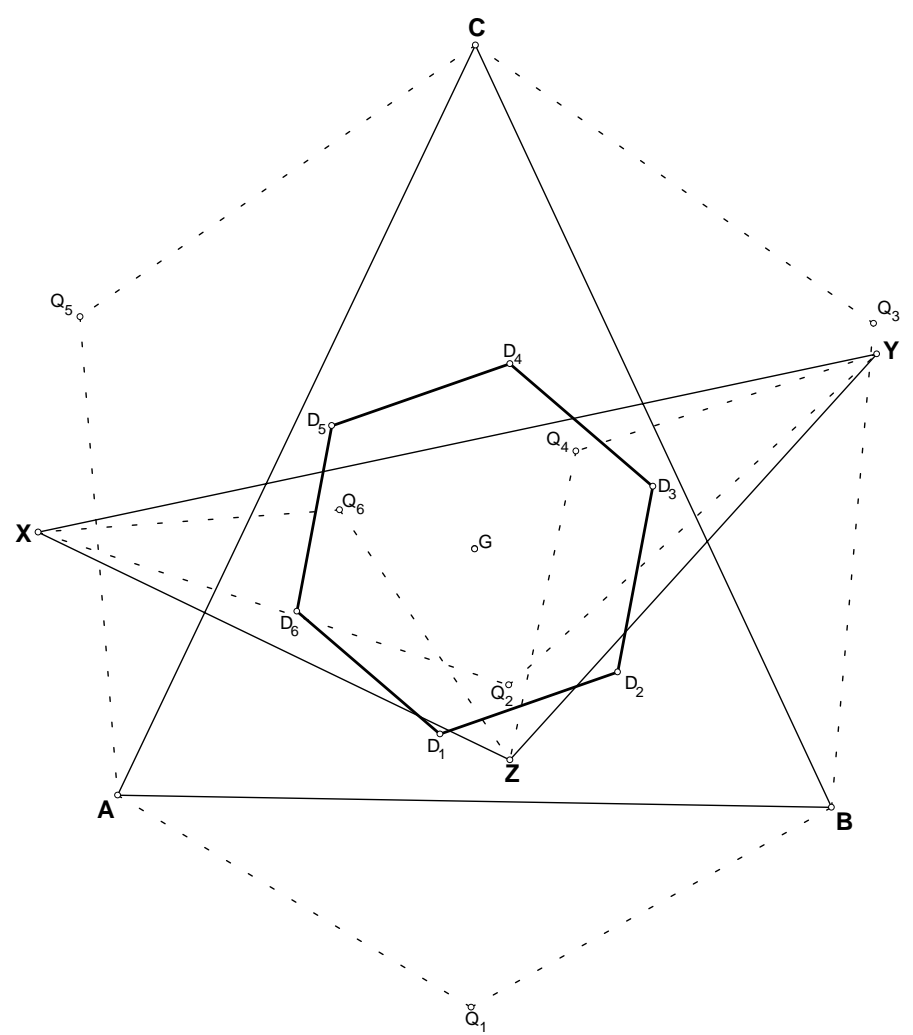

Fig. 3 In Theorem 5 we take centers of equilateral triangles on small diagonals of the hexagon AXBYCZ.

Proof. Let $\lambda=i \frac{\sqrt{3}}{3}, \mu=\frac{1}{2}(1+\lambda)$, and $\nu=\frac{1}{2}(1-\lambda)$. Under the same simplifications as in the proof of the Theorem 1, we can show that $Q_{1}=\mu A+\nu B, Q_{2}=\mu X+\nu Y$, $Q_{3}=-\nu A+\lambda B, Q_{4}=-\nu X+\lambda Y, Q_{5}=-\lambda A-\mu B$, and $Q_{6}=-\lambda X-\mu Y$. Also, one has

$$
\begin{array}{llrl}
D_{1} & =(\lambda A+\mu B+\mu X+\nu Y) / 3, & & D_{4}=-D_{1}, \\
D_{5}=(\nu A-\lambda B-\lambda X-\mu Y) / 3, & & D_{2}=-D_{5}, \\
D_{6}=(\mu A+\nu B+\nu X-\lambda Y) / 3, & \text { and } & D_{3}=-D_{6} .
\end{array}
$$

Notice that $\Omega \lambda=-\nu, \Omega \mu=\lambda$, and $\Omega \nu=\mu$. It is now obvious that $\Omega D_{1}=D_{2}$, $\Omega D_{2}=D_{3}, \Omega D_{3}=D_{4}, \Omega D_{4}=D_{5}, \Omega D_{5}=D_{6}$, and $\Omega D_{6}=D_{1}$. We conclude that the points $D_{1}, D_{2}, \ldots, D_{6}$ are vertices of a regular hexagon whose center is the common centroid of $A B C$ and $X Y Z$ because the rotation by $\frac{\pi}{3}$ around the origin maps each point $D_{i}$ to a point $D_{i+1}$ for $1 \leq i<6$ and $D_{6}$ to $D_{1}$.

In the above calculations we have again assumed that the triangle $\omega \Omega J$ is positively oriented and that we have constructed on small diagonals of the hexagon $P_{1} P_{2} \cdots P_{6}$ six positively oriented equilateral triangles. We obtain similar conclusions in the situation where negatively oriented triangles are used. 


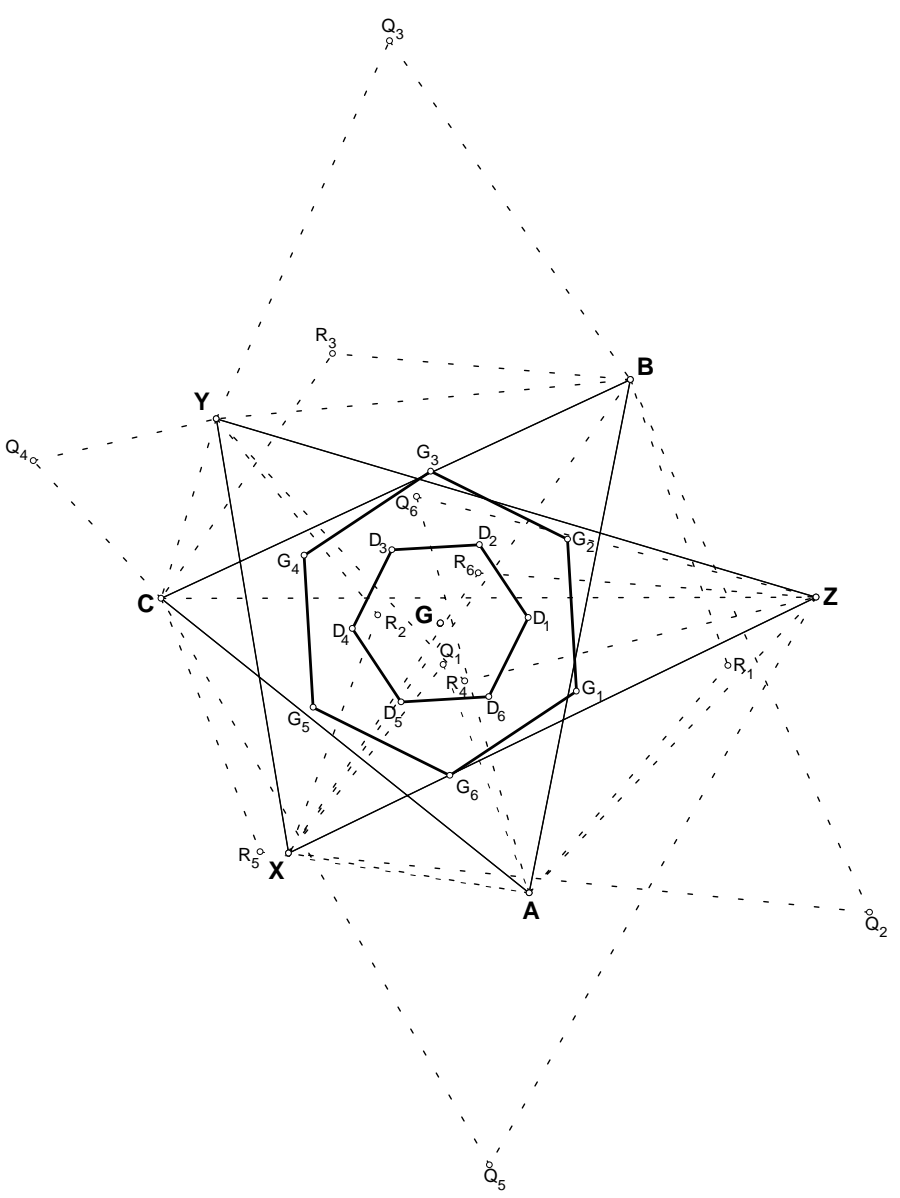

Fig. 4 Regular hexagons from Theorems 1 and 5 together.

We can now easily compute the lengths of sides of regular hexagons from Theorems 1 and 5 and check that their quotient is precisely $\sqrt{3}$.

\section{References}

[1] R. Deaux, Introduction to the geometry of complex numbers, Ungar Publishing Co., New York, 1956.

[2] L. Hahn, Complex numbers and geometry, Mathematical Association of America, Washington, 1994.

[3] Frank Morley and F.V. Morley, Inversive Geometry, Chelsea Publ. Co., New York, 1954.

[4] H. Schwerdtfeger, Geometry of complex numbers, Oliver and Boyd, Toronto, 1962.

[5] I.M. Yaglom, Complex numbers in geometry, Academic Press, New York, 1968.

Zvonko Čerin

Kopernikova 7

10010 Zagreb

Croatia 